\title{
Estudo experimental e de simulação CFD da dinâmica de partículas em tambores rotatórios com suspensores
}

\author{
S. M. NASCIMENTO ${ }^{1}$, G. A. S. CAMPOS ${ }^{1}$, D. M. LIMA ${ }^{1}$, L. F. G. ÁVILA ${ }^{1}$; M. A .S. BARROZO ${ }^{1}$, \\ C. R. DUARTE ${ }^{1}$. \\ ${ }^{1}$ Universidade Federal de Uberlândia, Faculdade de Engenharia Química \\ E-mail para contato: suellenmendonca01@gmail.com
}

\begin{abstract}
RESUMO - Os secadores rotatórios são equipamentos bastante utilizados devido à flexibilidade de operação e a elevada capacidade. Estes equipamentos consistem em tambores cilíndricos dotados de suspensores, que ao girarem promovem o cascateamento do sólido no interior do secador. Apesar da vasta aplicação do equipamento, ainda existe uma deficiência na modelagem do escoamento das partículas nos mesmos. Devido ao crescente aumento da capacidade de processamento dos computadores aliado ao alto custo das plantas piloto, a fluidodinâmica computacional (CFD) é uma ferramenta que tem sido cada vez mais utilizada no auxílio á compreensão do escoamento. Uma das abordagens do CFD é a abordagem Euler-Euler, que além de requerer um esforço computacional menor do que a Lagrangeana, permite fazer simulações mesmo em grandes escalas com um menor número de parâmetros. Quantificar o hold up nos suspensores é de extrema importância para a compreensão do escoamento, para isto, este trabalho foi pioneiro no desenvolvimento de uma metodologia que permite, através da análise de imagens, calcular a massa de sólidos nos suspensores do tambor rotatório, em função da posição angular, utilizando perfis de escoamento obtidos na simulação. Os resultados foram então comparados com ensaios experimentais. A modelagem utilizada na simulação foi adequada a esse processo e a metodologia desenvolvida se mostrou satisfatória para a utilização da abordagem Euler-Euler. Além disto, os resultados foram relevantes para discutir a natureza numérica do resolvedor FLUENT®.
\end{abstract}

\section{INTRODUÇÃO}

Os secadores rotatórios são equipamentos industriais utilizados principalmente na secagem de materiais granulados, apresentando uma grande capacidade de processamento e diversos tipos de aplicação. Segundo SHEEHAN et al. (2005), devido ao seu relativo baixo custo de operação, simplicidade de construção e flexibilidade para operar com diversos tipos de materiais, os secadores rotatórios são os mais utilizados dentre todos os tipos de secadores. O secador rotatório convencional consiste de uma armação cilíndrica, inclinada por um pequeno ângulo com relação à horizontal, que gira em torno do seu próprio eixo longitudinal. Além disso, acoplados internamente ao tambor existem suspensores que irão promover o cascateamento dos sólidos no interior do secador e melhorar o contato com o ar de secagem. 


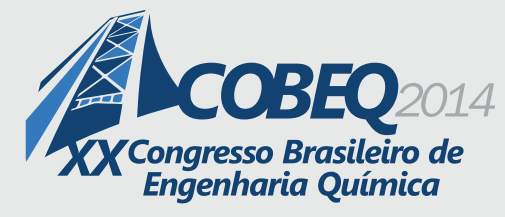

19 a 22 de outubro de 2014

Florianópolis/SC

Apesar da vasta aplicação destes secadores e da existência de trabalhos relevantes sobre secadores rotatórios, ainda existe uma deficiência em estudos sobre o comportamento da dinâmica das partículas nestes equipamentos. A compreensão dos perfis de descarga de sólidos dos suspensores é extremamente importante para a descrição do comportamento das partículas nos mesmos. Para isto, geralmente são utilizados tambores rotatórios com suspensores, sem a presença do ar de secagem, que facilitam as medidas da dinâmica das partículas nestes processos.

Alguns trabalhos experimentais já foram desenvolvidos com a finalidade de descrever o comportamento das partículas em secadores rotatórios. Porém, com o avanço da tecnologia e com a crescente capacidade de processamento dos computadores, aliados aos altos custos envolvidos na construção de plantas piloto, estão disponíveis novas ferramentas, como a fluidodinâmica computacional (CFD), que permite a abordagem de modelos matemáticos para a compreensão de fenômenos físicos. Entretanto, estudos de CFD devem ser validados experimentalmente.

De acordo com SANTOS et al. (2013), as abordagens Euler-Euler e Lagrange são as mais utilizadas para a simulação da dinâmica de materiais particulados em tambores rotatórios. $\mathrm{Na}$ abordagem lagrangeana, também conhecida como DEM (Discrete Element Method), as forças que atuam em cada partícula são calculadas baseadas nas leis de interação partícula-partícula enquanto que na abordagem Euler-Euler as diferentes fases são tratadas matematicamente como contínuas e interpenetrantes. Apesar de a simulação DEM ser amplamente utilizada para descrever o comportamento de partículas em tambores rotatórios com suspensores (GENG et al., 2009; GENG et al., 2011; GENG et al., 2013), nas simulações Euler-Euler o esforço computacional requerido é muito menor permitindo fazer simulações mesmo em grande escala com um menor número de parâmetros a ser definido.

Embora alguns autores já tenham utilizado a abordagem Euler-Euler para estudar o comportamento da queda de uma "cortina" de partículas através de uma corrente de ar (WARDJIMAN et al., 2008; AJAYI e SHEEHAN, 2012), , não existem trabalhos que utilizam esta abordagem para a simulação de tambor rotatório com suspensores. No presente trabalho desenvolveuse então o projeto de um tambor rotatório com suspensores, em escala reduzida e um coletor de partículas com a finalidade de determinar o hold-up, que é a massa de sólidos nos suspensores, em cada posição angular. Simultaneamente, foram feitas simulações utilizando a abordagem Euler-Euler com a finalidade de comparar os resultados obtidos com os dados experimentais. As simulações foram realizadas utilizando o software FLUENT ${ }^{\circledR}$ e este não permite obter o hold-up de forma direta. Para isso, também foi desenvolvida pelos autores uma metodologia que permite determinar a carga no suspensor em função da posição angular e comparar com os dados experimentais através dos perfis de escoamentos obtidos.

Assim, o objetivo do trabalho é comparar os valores de carga de sólidos nos suspensores em função da posição angular, obtidos através de trabalhos experimentais, com os valores estimados através de simulações via CFD, utilizando a abordagem Euler-Euler. 


\section{METODOLOGIA}

\subsection{Metodologia Experimental}

O equipamento utilizado no trabalho experimental consiste de um tambor cilíndrico com 0,108 $\mathrm{m}$ de diâmetro e $0,5 \mathrm{~m}$ de comprimento, fabricado em aço inox, colocado na horizontal. Na parte interna da casca do cilíndro existem seis suspensores igualmente espaçados com três segmentos cada. Cada segmento do suspensor possui o comprimento de 10,4 e $4 \mathrm{~mm}$, sendo que o maior segmento está soldado à casca cilíndrica. Na extremidade frontal do tambor, foi colocado um visor de vidro temperado que permite fotografar a posição angular do suspensor e na outra extremidade do tambor foi montado um sistema em acrílico com o coletor de partículas. A velocidade de rotação do tambor é medida utilizando um tacômetro digital.

Procedimento experimental: Adiciona-se a massa de partículas previamente determinada e inicia-se o movimento de rotação. Quando o suspensor em interesse atinge determinada posição angular, interrompe-se a rotação do cilindro e em seguida fotografa-se a posição angular do suspensor. Para medi-la, o tambor possui um transferidor, o qual permite que as retas horizontal e vertical sejam traçadas, como mostra a Figura 1. Utilizando o software Image J®, mede-se a posição angular, que é o ângulo formado pela reta que vai da ponta do suspensor até a origem e a reta horizontal. Posteriormente, retira-se a tampa da extremidade em acrílico e introduz-se o coletor de partículas. Realiza-se então a coleta das partículas, com o tambor inclinado para que não haja perda de massa, e mede-se a massa nesta posição.

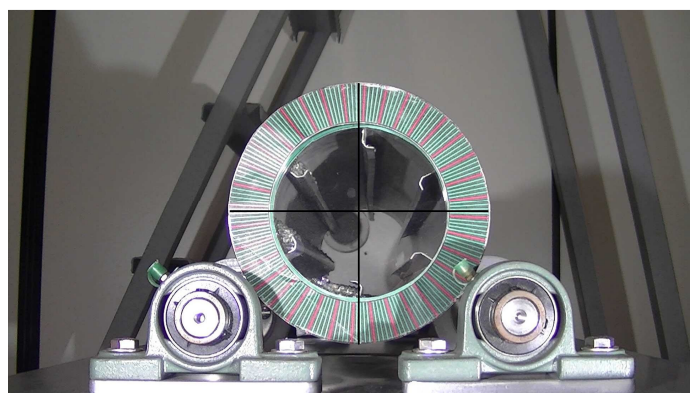

Figura 1 - Exemplo de foto utilizada para medir a posição angular da ponta do suspensor.

As partículas utilizadas no experimento foram esferas de vidro, que é um material inerte, com $2,6 \mathrm{~mm}$ de diâmetro e densidade de $2455 \mathrm{~kg} / \mathrm{m}^{3}$. A porosidade medida para essas partículas, para o leito empacotado, foi de 36,8\%. Utilizou-se cargas de partículas que correspondiam a 3 e $6 \%$ do volume do tambor. A velocidade de rotação utilizada foi de $3 \mathrm{rpm}$.

\subsection{Simulação Via CFD}

Modelagem: Utilizou-se o modelo Granular Euleriano Multifásico. A modelagem levou em consideração duas fases. Assim, o ar foi representado como primária, e as esferas de vidro como secundária. Para o cálculo da viscosidade granular utilizou-se o modelo de GIDASPOW (1994). 


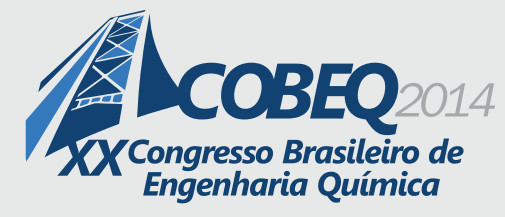

19 a 22 de outubro de 2014

Florianópolis/SC

Utilizou-se também, como parte da modelagem, a viscosidade friccional. Esta viscosidade atua como uma contribuinte à viscosidade cisalhante total e é originada a partir do atrito partícula-partícula (SANTOS, 2011). O modelo utilizado para a descrição do regime cisalhante friccional foi o modelo de Schaeffer, que é função do ângulo de fricção interno partícula-partícula. Este ângulo é obtido inclinando-se um plano com partículas coladas á superfície, e acima destas coloca-se partículas livres. Inicia-se o aumento da inclinação do plano e a partir do momento que as partículas livres começam a rolar, determina-se o ângulo de fricção interno (SANTOS, et al., 2013). Para as esferas de vidro deste trabalho, o ângulo de fricção interno utilizado foi de $28^{\circ}$.

Toda a modelagem detalhada do escoamento das partículas, assim como o equacionamento dos modelos utilizados, adotada neste trabalho pode ser encontrada no trabalho desenvolvido por SANTOS et al. (2013).

Condições de simulação utilizadas: Para que os dados de simulação pudessem ser comparados com os dados experimentais, as condições utilizadas foram as mesmas . Portanto, o diâmetro da fase secundária é de $2,6 \mathrm{~mm}$, a densidade de $2455 \mathrm{~kg} / \mathrm{m}^{3}$. O limite máximo de empacotamento medido é de $63,2 \%$ e velocidade de $3 \mathrm{rpm}$. Utilizaram-se diferentes condições de simulação. Primeiro, considerando que a fase secundária ocuparia $6 \%$ do volume do tambor, variou-se os limites de viscosidade friccional. Posteriormente, com o modelo simulado mais próximo dos resultados experimentais realizou-se uma simulação em que as partículas ocupariam 3\% do volume do tambor.

Determinação do hold-up nos suspensores: Através do hold-up, em função da posição angular, é possível descrever o perfil de descarga das partículas. Como o software FLUENT ${ }^{\circledR}$ não consegue de forma direta fornecer o valor da massa em determinado suspensor, foi necessário desenvolver uma metodologia que permitisse calculá-lo. Logo, este trabalho é pioneiro no que se trata á comparação quantitativa de uma simulação Euler-Euler de um tambor rotatório com suspensores e resultados experimentais.

Cada suspensor foi dividido em sub-volumes, para que a massa fosse calculada em cada um deles e posteriormente somada, desta maneira evitou-se que houvesse dúvidas a respeito da variação de massa ao longo do suspensor. Utilizando perfis de escoamento obtidos na simulação, em que cada cor representa uma fração de sólidos, foi possível através do software Image J® determinar a área correspondente a cada cor no suspensor para cada sub-volume. Esta área foi então multiplicada pela média da fração de sólidos para a cor correspondente. O resultado foi multiplicado pela densidade dos sólidos e pelo comprimento do sub-volume. Com a média de massa obtida pelos perfis das duas faces do sub-volume, obteve-se a massa deste. A massa total do suspensor é dada pela soma das massas de cada sub-volume. Para o cálculo da posição angular, assim como nas fotos do trabalho experimental, utilizou-se o software Image $\mathrm{J} \circledast$.

\section{RESULTADOS E DISCUSSÃO}

Através da metodologia desenvolvida, foi possível quantificar os resultados da simulação. Desta maneira foi possível estudar tanto a influencia do parâmetro viscosidade friccional, assim como a sensibilidade deste parâmetro aos limites em que ele passa a ser considerado. Na Figura 3 pode-se 


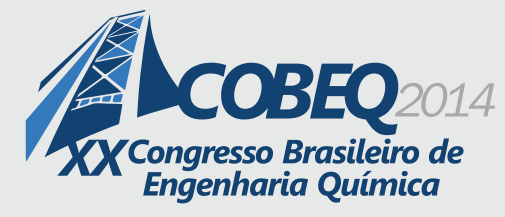

observar que todas as simulações seguem o resultado qualitativo obtido experimentalmente. Mas, quantitativamente, à medida que se diminui o limite para a consideração da viscosidade friccional a curva da simulação tende a se aproximar dos resultados experimentais. A diferença entre as curvas que possuem o limite de 50 e $60 \%$ é muito pequena, pois o volume de esferas que está entre estas frações é muito pequena. Observa-se também que estas duas curvas estão bem próximas da fração máxima de empacotamento do leito, $63,2 \%$, desta maneira não existe muita diferença entre estes resultados e a curva em que não é considerada a viscosidade friccional.

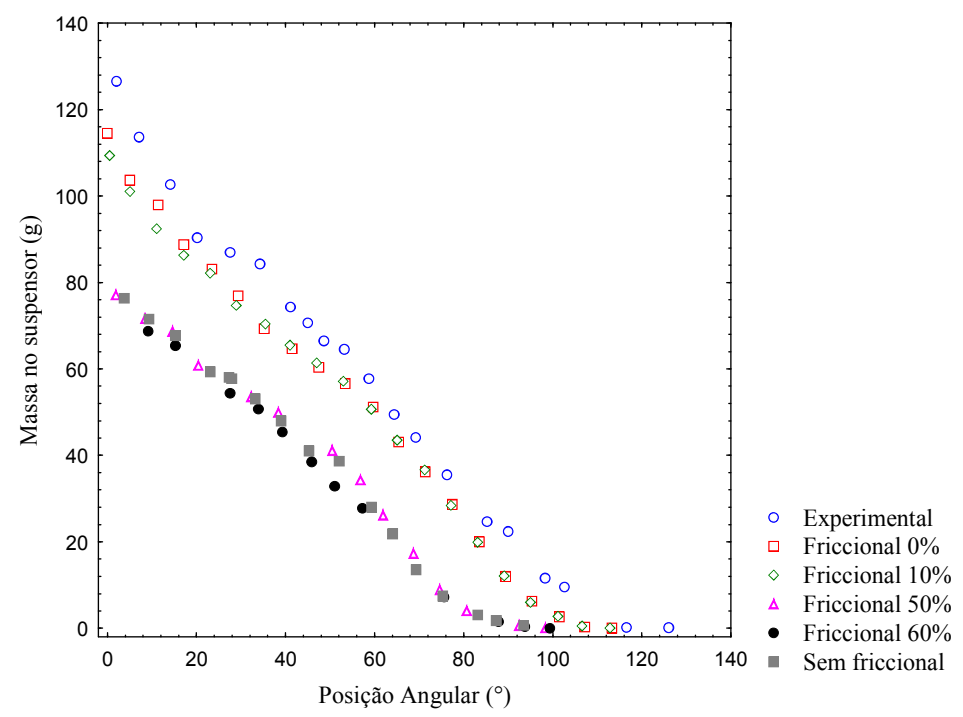

Figura 3 -Hold-up nos suspensores para diferentes parâmetros do modelo.

Assim como nos resultados para 50 e $60 \%$, as simulações que consideraram a viscosidade friccional para frações acima de $10 \%$ e para $0 \%$ ficaram bem próximas, sendo também justificado pela pequena quantidade de massa que está entre essas frações. No entanto, a curva para o limite inferior de $0 \%$ é a que melhor se ajustou ao experimento, principalmente para posições angulares mais baixas. Desta maneira, conclui-se que o modelo que melhor representa a dinâmica das partículas no tambor rotatório com suspensores é o que considera o regime friccional em qualquer região do leito $(0 \%)$.

De acordo com DARTEVELLE (2003), em seu trabalho sobre fluxos granulares, é usual considerar o regime friccional somente em leitos em que a concentração de sólidos é maior do que $50 \%$, contrastando com o resultado obtido. Este resultado abre a discussão sobre a característica de um software de resolução numérica, que é o caso do FLUENT ${ }^{\circledR}$. Experimentalmente, observa-se que não há diferença de frações de empacotamento das partículas no suspensor de acordo com a posição angular, como mostra a Figura 4. Quando se analisa o perfil de uma face obtido através da simulação é possível observar que ângulos que possuem uma posição angular mais alta possuem menores frações de partículas, enquanto que ângulos com posições mais baixas apresentam uma faixa de frações de partículas, que vão da mais concentrada até atingir a concentração nula. 

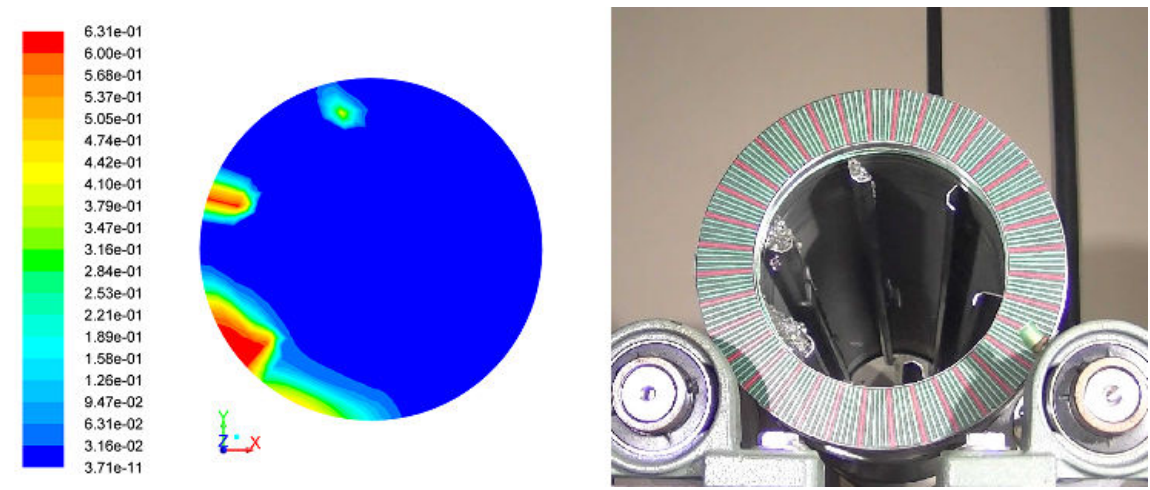

Figura 4 - Comparação dos perfis de porosidade simulado e experimental.

Como o FLUENT ${ }^{\circledR}$ é um resolvedor numérico de equações, numericamente é impossível que se passe da condição com uma fração de $63,2 \%$ de esferas para a condição em que não há partículas. Desta maneira, o software irá realizar uma interpolação, que pode ser verificada até mesmo no momento em que se inicializa o problema, como mostra a Figura 5:
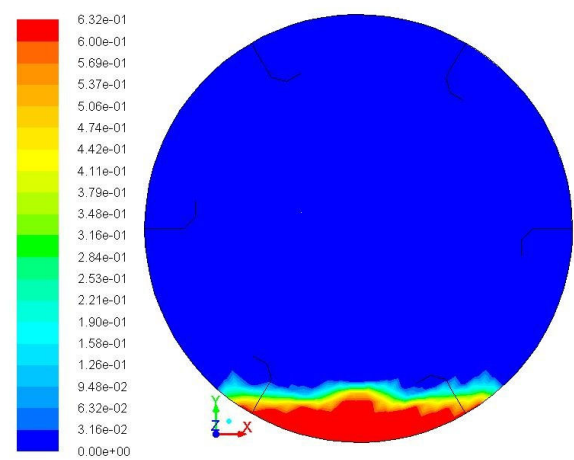

Figura 5 - Perfil de distribuição de esferas de vidro na face frontal do tambor na inicialização do problema.

$\mathrm{Na}$ Figura 5 a simulação foi inicializada com $6 \%$ do volume do tambor ocupado por esferas de vidro, assim, determinou-se que $1,2 \mathrm{~cm}$ de altura seriam ocupados pela fase secundária com uma fração de $63,2 \%$. Utilizando o software Image $\mathrm{J} 囚$, mediu-se a altura média ocupada pelas esferas e observou-se que é maior do que os $1,2 \mathrm{~cm}$ colocados inicialmente. Assim, para que a massa inicial colocada seja preservada, o software FLUENT ${ }^{\circledR}$ expande o volume do leito de partículas, o que se deve aos métodos de interpolação. No caso real, o empacotamento sai de um limite máximo para uma condição de empacotamento zero, mas numericamente é aplicada uma interpolação gerando valores intermediários, o que acarreta essa expansão do leito para que a massa seja preservada. Logo, é justificável, para esta simulação, que o regime friccional seja considerado a partir de $0 \%$, visto que experimentalmente verifica-se que até mesmo nas posições angulares mais altas a fração de empacotamento das partículas é alta. 


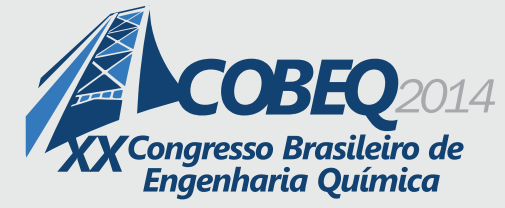

Com o objetivo de verificar a aplicação do modelo a outras condições, fez-se o trabalho experimental e a simulação com a condição em que apenas 3\% do volume do tambor é ocupado por esferas de vidro e a viscosidade friccional é aplicada em qualquer fração do leito. O gráfico que representa a variação de massa no suspensor de acordo com a posição angular, para $3 \%$ do volume do tambor ocupado por esferas de vidro, pode ser observado na Figura 6.

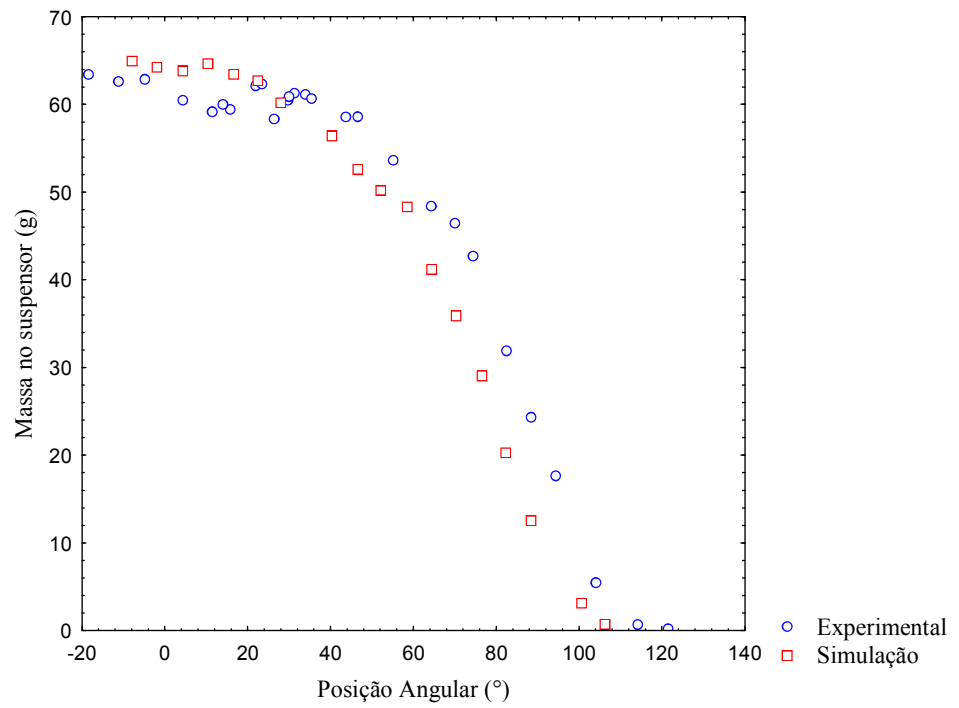

Figura 6 - Comparação dos valores de hold-up experimentais e simulado.

É possível analisar na Figura 6 que a simulação novamente conseguiu representar a variação do hold-up nos suspensores com a posição angular. Como para esta carga de partículas o tambor está operado abaixo da sua capacidade máxima, a descarga de sólidos começa somente depois da posição $0^{\circ}$ tanto no trabalho experimental quanto na simulação, e na região em que a massa permanece constante os resultados são bem próximos. Para posições angulares mais altas, em que os resultados para o tambor sobrecarregado e abaixo da sua capacidade são os mesmos, a mesma tendência da simulação com $6 \%$ é observada e a massa medida na simulação é um pouco menor do que a massa experimental.

\section{CONCLUSÃO}

De acordo com os resultados obtidos, conclui-se que a abordagem Euler-Euler foi uma ferramenta satisfatória para descrever o comportamento das partículas em um tambor rotatório com suspensores. Além disso, a metodologia desenvolvida se mostrou promissora em relação ao fato de quantificar o efeito dos limites de viscosidade friccional e em comparar com os dados experimentais. A modelagem da simulação, utilizando a viscosidade friccional para qualquer fração do leito, conseguiu prever os resultados experimentais para duas condições diferentes e se mostrou adequada ao caso. Este resultado chama atenção, também, ao fato do FLUENT ${ }^{\circledR}$ ser um resolvedor numérico de equações, que para preservar a massa realiza uma expansão do volume do leito para o cálculo das frações mássicas. Cabe ainda ressaltar o fato de muitas vezes esses resultados serem usados de forma 


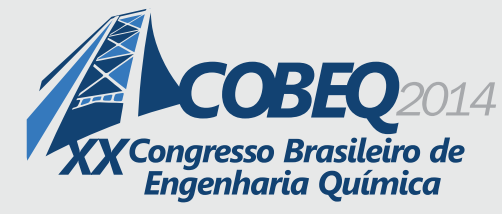

equivocada para a interpretação de perfis de escoamento e porosidade.

\section{AGRADECIMENTOS}

Os autores agradecem ao CNPQ, CAPES e FAPEMIG pelo apoio financeiro, e também agradece aos recursos concedidos no Projeto de Participação Coletiva em Eventos TécnicosCientíficos (PCE-00082-14) da FAPEMIG.

\section{REFERÊNCIAS BIBLIOGRÁFICAS}

AJAYI, O.O.; SHEEHAN, M.E. Application of image analysis to determine design loading in flighted rotary dryers. Powder Technology, v. 223, p. 123-130, 2012.

DARTEVELLE, S. Numerical and granulometric approaches to geophysical granular flows, Ph. D. thesis, Michigan Technological University, Department of Geological and Mining Engineering, Houghton, Michigan, 2003.

GENG, F.; YUAN, Z.; YAN, Y.; LUO, D.; WANG, H.; LI, B.; XU, D. Numerical simulation on mixing kinetics of slender particles in a rotary dryer. Powder Technology, v. 193, p. 50-58, 2009.

GENG. F.; LI, Y.; WANG, X.; YUAN, Z.; YAN, Y.; LUO, D. Simulation of dynamic processes on flexible filamentous particles in the transverse section of a rotary dryer and its comparison with ideo-imaging experiments. Powder Technology, v. 207, p. 175-182, 2011.

GENG, F.; LI, Y.; YUAN, L.; LIU, M.; WANG, X.; YUAN, Z.; YAN, Y.; LUO, D. Experimental study of the space time of flexible filamentous particles in a rotary dryer. Exp. Thermal and Fuid Sci. v. 44. p. 708-715, 2013.

GIDASPOW, D. Multiphase flow and fluidization: Continuum and Kinetic Theory Descriptions, California: Academic Press, INC., 1994.

SANTOS, D.A. Contribuições ao estudo da fluidodinâmica em leito de jorro- Estudos experimentais e de simulação via CFD. Dissertação de mestrado. PPGEQ/UFU- Uberlândia/MG, 106 p., 2011.

SANTOS, D.A.; PETRI, I.J.; DUARTE, C.R.; BARROZO, M.A.S. Experimetal and CFD study of the hydrodynamic behavior in a rotating drum. Powder Technology, v. 250, p. 52-62, 2013.

SHEEHAN, M.E.; BRITTON, P.F.; SCHNEIDER, P.A. A model for solids transport in flighted rotary dryers based on physical considerations. Chem. Eng. Sci. v.60, p. 4171-4182, 2005.

WARDJIMAN, C.; LEE ,A.; RHODES, M. Behaviour of a curtain of particles falling through a horizontally-flowing gas stream. Powder Technology, v. 188, p. 110-118, 2008. 